\title{
Ten-Year Prevalence Trends of Phenotypically Identified Community-Associated Methicillin-Resistant Staphylococcus aureus Strains in Clinical Specimens
}

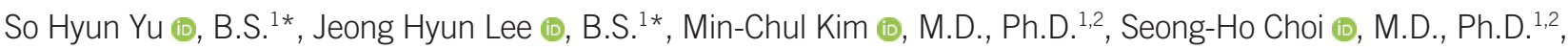

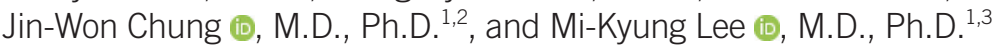 \\ ${ }^{1}$ Chung-Ang University College of Medicine, ${ }^{2}$ Division of Infectious Diseases, Department of Internal Medicine, ${ }^{3}$ Department of Laboratory Medicine, \\ Chung-Ang University Hospital, Seoul, Korea
}

\begin{abstract}
Background: Community-associated methicillin-resistant Staphylococcus aureus (CA-MRSA) strains were first detected in hospitals in Korea between the late 2000s and early 2010s. However, there is limited information regarding the prevalence of CA-MRSA strains among hospital isolates and their phenotypic changes over the last decade. We investigated the prevalence trend of CA-MRSA strains isolated from different clinical specimens and their phenotypic changes between September 2009 and September 2019.
\end{abstract}

Methods: CA-MRSA strains were phenotypically identified by confirming their resistance to penicillin (PCN) and oxacillin (OXA) and evaluating their susceptibility to trimethoprimsulfamethoxazole, rifampin, fusidic acid, tetracycline, and at least one of the following four antimicrobials: clindamycin (CLI), erythromycin (ERY), ciprofloxacin (CIP), and gentamicin (GEN). A CA-MRSA strain that exhibited resistance to ERY, CLI, CIP, or GEN was classified as having resistance pattern I, II, III, or IV, respectively, regardless of its resistance to other antimicrobial agents.

Results: Of the 8,278 MRSA isolates identified in specimens obtained two days after admission, 1,385 (16.73\%) were CA-MRSA strains. The prevalence of CA-MRSA strains increased from $12.2 \%$ to $26.6 \%$ (3.21\% per period, $P=0.05$ ). Resistance type analysis revealed an increasing trend in the prevalence of PCN/OXA-resistant (1.84\%; $P=0.049$ ) and PCN/OXA/ERY/CLI/CIP-resistant $(0.98 \% ; P=0.04)$ CA-MRSA strains and in resistance pattern III strains (2.08\%; $P=0.004)$.

Conclusions: The prevalence of CA-MRSA strains in Korea has increased significantly over the last decade, and CA-MRSA strains have gained phenotypic diversity beyond PCN/OXAresistance, including antimicrobial resistance to non- $\beta$-lactams, especially CIP.

Key Words: Community-associated methicillin-resistant Staphylococcus aureus, Prevalence, Phenotypic changes, Antimicrobial resistance
Received: June 29, 2020

Revision received: September 10, 2020

Accepted: January 11, 2021

\section{Corresponding author:}

Seong-Ho Choi, M.D., Ph.D.

Division of Infectious Diseases, Department of Internal Medicine, Chung-Ang University Hospital, 102 Heukseok-ro, Dongjak-gu,

Seoul 06973, Korea

Tel: +82-2-6299-3142

Fax: +82-2-6299-2064

E-mail: tobeservant@cau.ac.kr

*These authors equally contributed to this study.

\section{(i) \$}

(c) Korean Society for Laboratory Medicine

This is an Open Access article distributed under the terms of the Creative Commons Attribution Non-Commercial License (https://creativecommons.org/licenses/by-nc/4.0) which permits unrestricted non-commercial use, distribution, and reproduction in any medium, provided the original work is properly cited.

\section{INTRODUCTION}

Methicillin-resistant Staphylococcus aureus (MRSA) is one of the leading causative agents of hospital-acquired infections worldwide [1]. Community infection due to MRSA, first reported in the late 1990s, is referred to as community-associated MRSA (CAMRSA) infection. CA-MRSA has posed a significant public health challenge; it was initially detected in North America and Europe and subsequently in Korea in the mid-2000s [2, 3]. Unlike MRSA strains responsible for hospital-acquired infections, CA-MRSA 
strains are mostly susceptible to non- $\beta$-lactam antimicrobials owing to their small, resistance-conferring genomic complex (staphylococcal cassette chromosome mec type IV [SCCmec type IV]) that does not harbor resistance genes against non- $\beta$ lactams [2]. The prevalence of CA-MRSA strains has increased significantly since their detection, and these strains have recently spread to hospitals. In the United States, infections caused by USA300, a major CA-MRSA strain, have increased in hospitals, and serious infections caused by this strain, such as $S$. aureus bacteremia, even have altered clinical characteristics [4, 5]. Several studies have shown that infections caused by multilocus sequence typing (MLST) sequence type 72 (ST72), a major CAMRSA strain in Korea, are increasing in hospitals in Korea compared with the late 2000s to early 2010s [6-8]. However, followup studies on the prevalence of CA-MRSA strains in hospitals after this period are limited. These strains have rarely been investigated using different types of clinical specimens; hence, further changes in the antimicrobial resistance patterns of CAMRSA strains in hospitals need to be studied. The lack of relevant reports could be attributed to the fact that it is quite challenging to conduct genotyping experiments to differentiate CAMRSA strains from hospital MRSA strains among numerous MRSA isolates obtained from various clinical specimens over a long period. Therefore, we phenotypically identified CA-MRSA strains based on previous studies [9-22] and antimicrobial resistance test (ART) results of isolates from various clinical specimens. Subsequently, we investigated the prevalence of and phenotypic changes in the CA-MRSA strains identified.

\section{MATERIALS AND METHODS}

\section{Collection of study specimens}

This retrospective study used reports of clinical S. aureus specimens identified using bacterial culture tests at Chung-Ang University Hospital, an 850-bed tertiary care center in Seoul, Korea, over the last 10 years (September 2009 to September 2019). The study was divided into five periods: period 1 (September 2009 to August 2011), period 2 (September 2011 to August 2013), period 3 (September 2013 to August 2015), period 4 (September 2015 to August 2017), and period 5 (September 2017 to September 2019). If a specimen was collected in the outpatient or emergency department or the interval between the date of admission and the date of specimen collection was less than two days, the infection was considered community-associated (CA). If the interval exceeded two days, it was considered hospital-acquired. During the study period, S. aureus strains were isolated from 16,048 clinical specimens, including 8,271 sputum (51.54\%), 1,917 ear-nose-throat (ENT; 11.95\%), 1,867 blood (11.63\%), 755 urogenital (urine or vaginal discharge; $4.70 \%$ ), 458 abscess fluid (2.85\%) specimens, and 2,780 specimens from other sources (17.32\%). Of these isolates, 11,266 (70.20\%) were identified as MRSA; 1,527 (13.55\%) were collected from community settings, and 9,739 (86.45\%) were collected from hospital settings. Of the 9,739 hospital-acquired MRSA isolates, 8,278 (85.00\%) obtained from clinically important specimens, such as sputum $(\mathrm{N}=6,091)$, blood $(\mathrm{N}=966)$, ENT ( $N=635)$, urogenital $(N=371)$, and abscess fluid $(N=215)$ specimens, were ultimately included in this study.

\section{Definition of phenotypically identified CA-MRSA strains and their phenotypes}

The phenotypic identification of CA-MRSA strains was based on the ART results of CA-MRSA strains previously reported in Korea (Table 1). Antimicrobial resistance to trimethoprim-sulfamethoxazole, rifampin, fusidic acid, and tetracycline has rarely been observed in Korean CA-MRSA strains; however, the resistance rates to erythromycin (ERY), clindamycin (CLI), ciprofloxacin (CIP), and gentamicin (GEN) ranged between $20.12 \%$ and 41.50\% [9-22]. Therefore, the CA-MRSA strain phenotype was defined based on the following criteria: (1) resistance to penicillin (PCN) and oxacillin (OXA); (2) susceptibility to trimethoprimsulfamethoxazole, rifampin, fusidic acid, and tetracycline; and (3) susceptibility to at least one of the following four non- $\beta$-lactams: ERY, CLI, CIP, and GEN. An isolate that satisfied the first two conditions and was resistant to all four non- $\beta$-lactams was not categorized as a CA-MRSA strain, because it was difficult to distinguish it from hospital-acquired MRSA strains. Based on our definition, MLST ST72 was considered a CA-MRSA strain, as its ART results (Supplemental Data Table S1) are similar to those of the isolates from previous studies listed in Table 1. The CA-MRSA strains were further classified into 15 categories based on the ART results; of these, the four major CA-MRSA phenotypes were PCN/OXA-resistance, PCN/OXA/CLI-resistance, PCN/OXA/CIPresistance, and PCN/OXA/CLI/CIP-resistance. A CA-MRSA strain that was resistant to ERY, CLI, CIP, or GEN was classified as having resistance pattern I, II, III, or IV, respectively, regardless of its resistance to other antimicrobial agents (Table 2).

The study was exempted from review by the Institutional Review Board of Chung-Ang University Hospital, because no patient-identifiable information was collected and the data were analyzed anonymously. 
Table 1. Antimicrobial resistance patterns of CA-MRSA clinical isolates in Korea

\begin{tabular}{|c|c|c|c|c|c|c|c|c|c|}
\hline \multirow{2}{*}{ Reference } & \multirow{2}{*}{ Total (N) } & \multicolumn{8}{|c|}{ Antimicrobial resistance (N) } \\
\hline & & ERY & CLI & CIP & GEN & Fusidic acid & TET & RIF & TMP_SMX \\
\hline Jo0, et al. 2012 [9] & 84 & 36 & 21 & & & 2 & & & \\
\hline Shin, et al. 2016 [10] & 36 & 16 & 0 & 1 & 11 & & 5 & & \\
\hline Lee, et al. 2011 [11] & 29 & 18 & 18 & & & & 0 & & \\
\hline Ko, et al. 2008 [12] & 18 & 12 & 7 & 3 & 9 & & 3 & 0 & \\
\hline Sung, et al. 2012 [13] & 40 & 24 & 8 & 7 & 14 & & 7 & 1 & 1 \\
\hline Kim, et al. 2008 [14] & 25 & 14 & 14 & 0 & 2 & & 1 & 0 & 0 \\
\hline Peck, et al. 2009 [15] & 36 & 32 & 30 & 30 & 30 & & 26 & 2 & 3 \\
\hline Bae, et al. 2010 [16] & 42 & 25 & 2 & 3 & 6 & & 3 & 0 & 1 \\
\hline Moon, et al. 2010 [17] & 2 & 1 & 1 & 0 & 0 & & 0 & 0 & 0 \\
\hline Kwon, et al. 2011 [18] & 58 & & & 44 & & & 39 & & \\
\hline Park, et al. 2015 [19] & 79 & 28 & 18 & 5 & 12 & 0 & & 0 & 0 \\
\hline Bae, et al. 2019 [20] & 16 & 4 & 4 & 4 & 4 & 2 & 1 & & \\
\hline Choi, et al. [22] (Unpublished data) & 299 & 83 & 61 & 34 & 47 & 8 & 9 & 6 & 2 \\
\hline Overall resistance, $\mathrm{N}(\%)$ & 764 & $\begin{array}{c}293 / 706 \\
(41.50)\end{array}$ & $\begin{array}{c}229 / 764 \\
(29.97)\end{array}$ & $\begin{array}{c}131 / 651 \\
(20.12)\end{array}$ & $\begin{array}{c}135 / 593 \\
(22.77)\end{array}$ & $\begin{array}{c}12 / 478 \\
(2.51)\end{array}$ & $\begin{array}{l}94 / 601 \\
(15.64)\end{array}$ & 9/597 (1.51) & $7 / 579(1.21)$ \\
\hline
\end{tabular}

Abbreviations: CA-MRSA, community-associated methicillin-resistant Staphylococcus aureus; CLI, clindamycin; ERY, erythromycin; CIP, ciprofloxacin; GEN, Gentamycin; TET, Tetracycine; RIF, Rifampicin; TMP_SMX, trimethoprim/sulfamethoxazole.

Table 2. Phenotypes of CA-MRSA strains among HA-MRSA isolates

\begin{tabular}{|c|c|c|c|c|c|c|c|}
\hline \multirow{2}{*}{ Type } & \multicolumn{6}{|c|}{ Results of antimicrobial resistance tests } & \multirow{2}{*}{ Isolates (N) } \\
\hline & PCN/OXA & ERY & $\mathrm{CLI}$ & CIP & GEN & Others & \\
\hline 1 & $\mathrm{R}$ & S & S & S & S & S & 697 \\
\hline 2 & $\mathrm{R}$ & $\mathrm{R}$ & $S$ & $S$ & S & S & 6 \\
\hline 3 & $\mathrm{R}$ & S & $\mathrm{R}$ & $S$ & S & S & 2 \\
\hline 4 & $\mathrm{R}$ & S & S & $\mathrm{R}$ & S & S & 86 \\
\hline 5 & $\mathrm{R}$ & S & $S$ & $S$ & $\mathrm{R}$ & $S$ & 34 \\
\hline 6 & $\mathrm{R}$ & $\mathrm{R}$ & $\mathrm{R}$ & $S$ & S & $S$ & 241 \\
\hline 7 & $\mathrm{R}$ & $R$ & $S$ & R & S & S & 107 \\
\hline 8 & $\mathrm{R}$ & $R$ & $S$ & $S$ & $\mathrm{R}$ & $S$ & 0 \\
\hline 9 & $\mathrm{R}$ & S & R & $\mathrm{R}$ & S & S & 0 \\
\hline 10 & $\mathrm{R}$ & S & $\mathrm{R}$ & S & $\mathrm{R}$ & S & 0 \\
\hline 11 & $\mathrm{R}$ & S & S & $\mathrm{R}$ & $\mathrm{R}$ & S & 0 \\
\hline 12 & $\mathrm{R}$ & $R$ & $\mathrm{R}$ & $\mathrm{R}$ & S & S & 177 \\
\hline 13 & $\mathrm{R}$ & $\mathrm{R}$ & $\mathrm{R}$ & S & $\mathrm{R}$ & S & 10 \\
\hline 14 & $\mathrm{R}$ & $\mathrm{R}$ & S & $\mathrm{R}$ & $\mathrm{R}$ & S & 0 \\
\hline \multirow[t]{2}{*}{15} & $\mathrm{R}$ & $S$ & $\mathrm{R}$ & $\mathrm{R}$ & $\mathrm{R}$ & S & 0 \\
\hline & & $\begin{array}{l}\text { Resistance pattern } \\
\qquad I^{*}(\mathrm{~N}=541)\end{array}$ & $\begin{array}{l}\text { Resistance pattern } \\
\qquad \|^{\dagger}(\mathrm{N}=430)\end{array}$ & $\begin{array}{l}\text { Resistance pattern } \\
\qquad \|^{\ddagger}(N=370)\end{array}$ & $\begin{array}{l}\text { Resistance pattern } \\
\quad I \mathbf{V}^{\S}(\mathrm{N}=44)\end{array}$ & & \\
\hline
\end{tabular}

* Resistance pattern I (ERY-R phenotypically identified CA-MRSA strains). ${ }^{\dagger}$ Resistance pattern II (CLI-R phenotypically identified CA-MRSA strains). ${ }^{\ddagger}$ Resistance pattern III (CIP-R phenotypically identified CA-MRSA strains). ${ }^{\S}$ Resistance pattern IV (GEN-R phenotypically identified CA-MRSA strains).

Abbreviations: CA-MRSA, community-associated methicillin-resistant Staphylococcus aureus; HA-MRSA, hospital-acquired methicillin-resistant S. aureus; S, susceptible; R, resistant; PCN, penicillin; OXA, oxacillin; ERY, erythromycin; CLI, clindamycin; CIP, ciprofloxacin; GEN, gentamicin. 
Yu SH, et al.

Ten-year study of CA-MRSA strains

\section{Antimicrobial resistance testing}

ART was conducted using Vitek 2 AST-P601 cards (bioMérieux, Inc., Marcy-l'Étoile, France) and MIC breakpoints according to the CLSI document M100 [23].

\section{Statistical analysis}

Statistical analysis was performed to evaluate the proportions of phenotypic changes among the CA-MRSA strains over the study period. Linear regression analysis was performed to determine the degree of change in the prevalence per period and whether
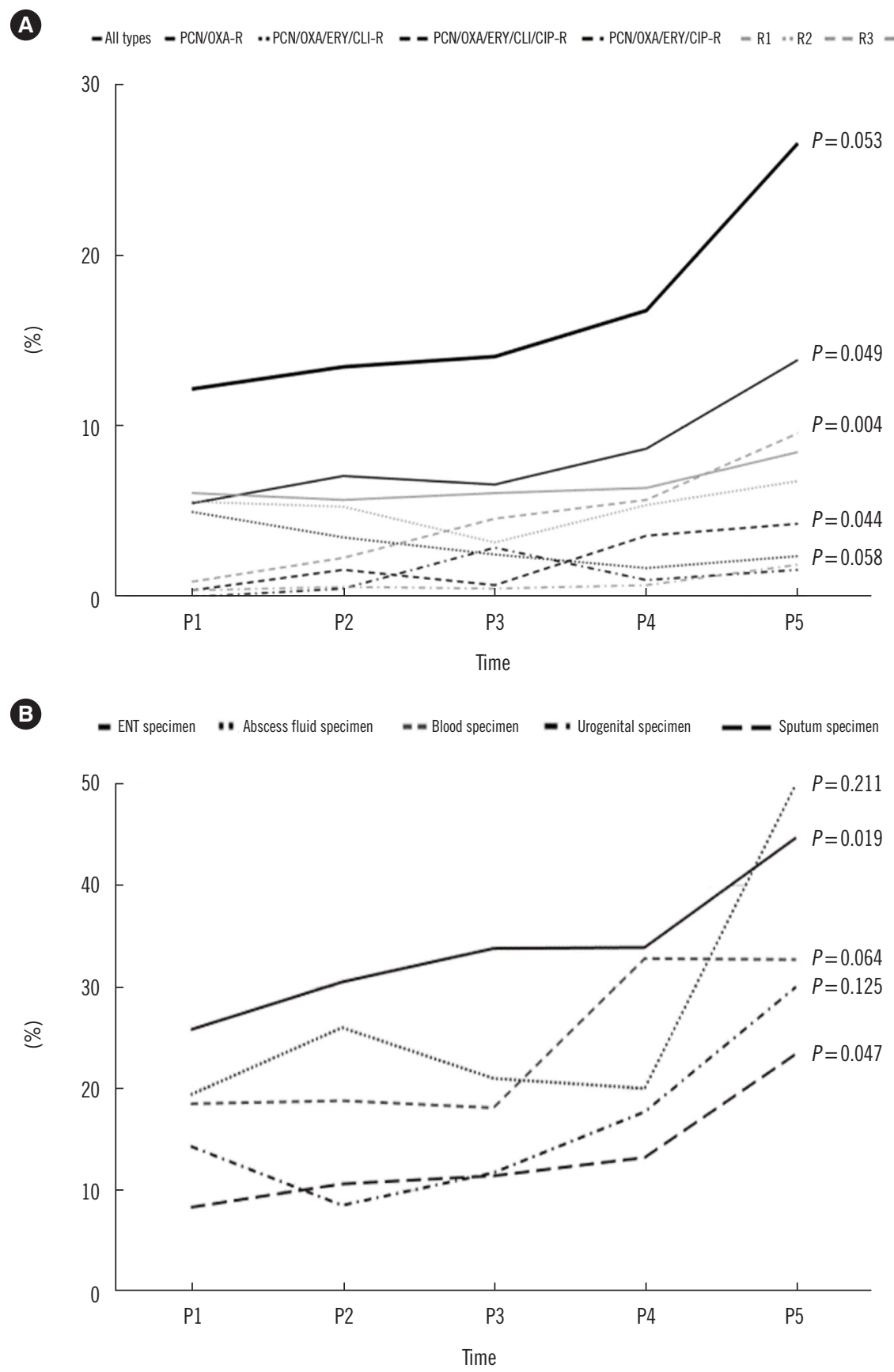

Fig. 1. Prevalence trends of CA-MRSA strains according to resistance type (A) and specimen type (B), of PCN/OXA-resistant (C) and resistance pattern III (D) CA-MRSA strains according to specimen type, and of PCN/OXA/ERY/CLI/CIP-resistant CA-MRSA strains (E) according to specimen type, among HA-MRSA isolates.

Abbreviations: MRSA, methicillin-resistant Staphylococcus aureus; CA-MRSA, community-associated MRSA; PCN, penicillin; OXA, oxacillin; ERY, erythromycin; CLI, clindamycin; CIP, ciprofloxacin; HA-MRSA, hospital-acquired MRSA; R1-4, resistance pattern I-IV; ENT, ear-nose-throat.

(Continued to the next page) 

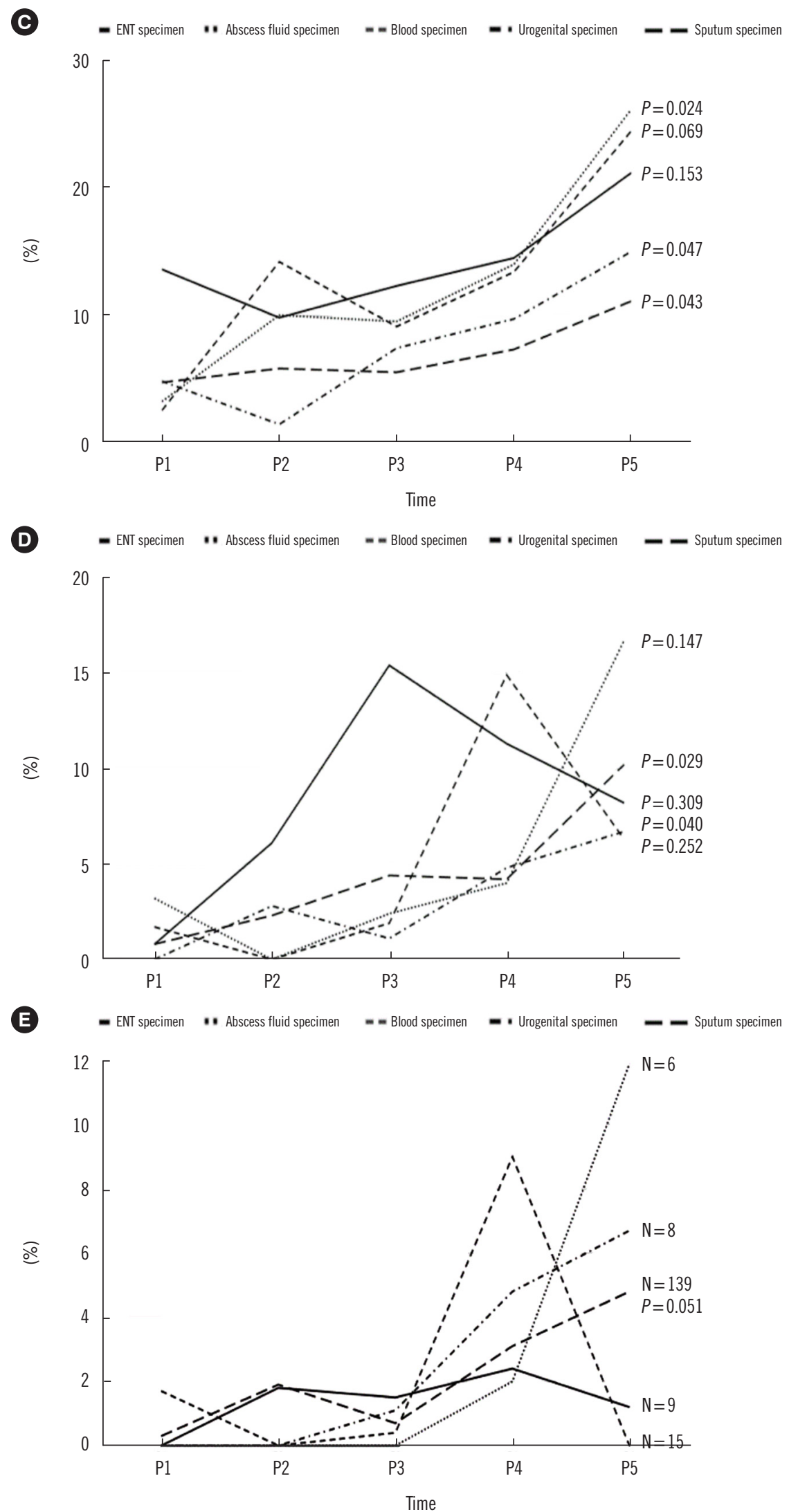

Fig. 1. Continued. 
the increasing or decreasing trend was statistically significant. Statistical analyses were performed using the SPSS software version 18.0 (SPSS Inc., Chicago, IL, USA), and $P<0.05$ was considered statistically significant.

\section{RESULTS}

Of the 8,278 isolates investigated, 1,385 (16.73\%) were phenotypically categorized as CA-MRSA strains. CA-MRSA strains were most commonly detected in ENT specimens (208/635, 32.76\%), followed by abscess fluid (59/215, 27.44\%), blood (227/966, $23.50 \%)$, urogenital (58/371, 15.63\%), and sputum (833/6,091, $13.68 \%)$. The most frequently isolated CA-MRSA strain phenotypes were PCN/OXA-resistance ( $N=697)$, PCN/OXA/ERY/CLIresistance $(\mathrm{N}=241), \mathrm{PCN} / \mathrm{OXA} / \mathrm{ERY} / \mathrm{CLI} / \mathrm{CIP}$-resistance $(\mathrm{N}=177)$, and PCN/OXA/ERY/CIP-resistance $(\mathrm{N}=107)$ and $541,430,370$, and 44 isolates exhibited resistance patterns I, II, III, and IV, respectively (Table 2 ).

The prevalence of CA-MRSA strains among hospital-acquired MRSA isolates showed an increasing trend from $12.2 \%$ (period 1) to $26.6 \%$ (period 5), with an increase of $3.21 \%$ per period $(P=0.053)$. When analyzed by resistance type, an increasing trend was observed in the proportions of PCN/OXA-resistant (increase per period, 1.84\%; $P=0.049$ ) and PCN/OXA/ERY/CLI/ CIP-resistant CA-MRSA strains (increase per period, 0.98\%; $P=0.044$ ) as well as in resistance pattern III strains (increase per period, 2.08\%; $P=0.004$ ). However, the proportion of PCN/ OXA/ERY/CLI-resistant CA-MRSA strains showed a decreasing trend (decrease per period, $0.70 \% ; P=0.058$; Fig. $1 A$ ). An increasing trend was observed in the proportions of CA-MRSA strains in the ENT (increase per period, $4.12 \% ; P=0.019$ ), sputum (increase per period, 3.28\%; $P=0.047$ ), and blood (increase per period, 4.24\%; $P=0.064$ ) specimens (Fig. 1B).

The increasing trend in the proportion of PCN/OXA-resistant CA-MRSA strains was observed in all specimen types [abscess fluid (increase per period, 5.00\%; $P=0.024$ ), sputum (increase per period, $1.43 \% ; P=0.043$ ), urogenital (increase per period, 2.87\%; $P=0.047$ ), and blood (increase per period, $4.32 \% ; P=$ 0.069) specimens], except ENT specimens (increase per period, 1.99\%; $P=0.153$; Fig. 1C). An increasing trend in the proportion of resistance pattern III strains was observed in sputum (increase per period, 2.07\%; $P=0.029$ ) and urogenital (increase per period, $1.54 \% ; P=0.040$ ) specimens (Fig. 1D). The majority of PCN/OXA/ERY/CLI/CIP-resistant CA-MRSA strains were isolated from sputum specimens (139 of $177,78.53 \%$ ), and an increasing trend (increase per period, $1.02 \% ; P=0.051$ ) in the proportion of these strains was also observed in sputum specimens (Fig. 1E).

\section{DISCUSSION}

The proportion of CA-MRSA strains among hospital-acquired MRSA isolates obtained from a tertiary care center showed an increasing trend over the last decade. This trend appears to have been caused by an increase in the prevalence of PCN/OXA-resistant CA-MRSA strains and various types of CIP-resistant CAMRSA strains.

Clinical studies on CA-MRSA strains were first conducted in the late 2000s in Korea, mostly using blood and nasal specimens. A number of studies involving blood specimens reported that the CA-MRSA genotype accounted for $10-20 \%$ of isolates in hospital-acquired infections [17, 18, 21]. This is consistent with our results wherein phenotypically identified CA-MRSA strains accounted for $18.5 \%$ of hospital-acquired MRSA blood isolates during the period 1 (Fig. 1B). However, to date, no study has examined changes in the prevalence of CA-MRSA strains among hospital isolates using multiple specimens, such as abscess fluid, sputum, and urogenital specimens. To the best of our knowledge, this is the first study to phenotypically characterize CA-MRSA strains obtained from various clinical specimens to obtain crucial information regarding the prevalence of the CA-MRSA genotype in hospitals over a 10-year period.

We observed that the prevalence of CA-MRSA strains doubled from $12.20 \%$ to $26.60 \%$ in the last 10 years. This increasing trend was also observed for ENT, blood, and sputum specimens (Fig. 1B). Although a notably increasing trend in the prevalence of CA-MRSA strains was observed in abscess fluid and urogenital specimens (Fig. 1B) as well, this trend was not statistically significant, as the numbers of CA-MRSA strains detected in abscess fluid and urogenital specimens were considerably low (59 and 58, respectively). Taken together, this study revealed (based on indirect evidence) that the prevalence of CA-MRSA strains is increasing in Korean hospitals. Additionally, CA-MRSA strains were most commonly detected in ENT specimens, which is consistent with a report indicating that $50 \%$ of MRSA strains detected in nasal specimens of newly admitted patients in the late 2000s were of the ST72 genotype [8]. Our results differ from those of contemporary studies mainly conducted using blood specimens in that the proportion of CA-MRSA isolates found was not as high as previously reported [17, 18, 21]. The high proportion of CA-MRSA strains identified in ENT specimens suggests that the CA-MRSA genotype was initially introduced into 
hospitals through nasal carriage, with subsequent infection of other sites in the human body.

The ART pattern of ST72, which is the most prevalent CAMRSA genotype, is known to be identical to that of the phenotypically identified PCN/OXA-resistant CA-MRSA strains [7]. Resistance to ERY, CLI, CIP, and GEN has been rarely reported in ST72 genotype strains [7]. Unexpectedly, we observed an increase in the proportion of PCN/OXA/ERY/CLI/CIP-resistant CA$M R S A$, along with an increased number of strains with resistance pattern III during the study period. The increase in CIP resistance is considered a major contributor to the increase in the proportion of PCN/OXA/ERY/CLI/CIP-resistant strains with the decrease in the proportion of PCN/OXA/ERY/CLI-resistant strains. This result is also consistent with an increased proportion of resistance pattern III strains. These results indicate that the prevalence of CA-MRSA strains with CIP resistance is increasing with the overall increase in the prevalence of ST72 strains (PCN/OXA-resistant CA-MRSA), indicating increasing genotypic diversity of CA-MRSA strains. When the increase in resistance pattern III strains was analyzed according to specimen types, the prevalence of CIP-resistant strains significantly increased among isolates from sputum and urogenital specimens. This indicates that the proportion of CIP-resistant strains first increased in the community environment owing to the frequent use of CIP for the treatment of community-acquired pneumonia and urinary tract infections; subsequently, these strains were introduced into hospitals. Data from a recent study (unpublished) on a large prospective cohort of adult patients with $S$. aureus bacteremia conducted at a tertiary care center showed that the ST72/spa-t148 and ST8 genotypes exhibited greater CIP resistance than did other spa types of ST72 [22]. Therefore, it is necessary to determine whether the proportions of specific ST72 and ST8 spa types, including USA300, is increasing in the Korean hospital environment.

This study had a few significant limitations. First, we did not genotype the MRSA isolates in this study. Although phenotypic identification of CA-MRSA may not be as accurate as genotypic identification, collecting MRSA isolates from clinical specimens over a long study period followed by genotyping can be considerably challenging. However, the study design adopted in this study is advantageous in that it sheds light on the changes in the prevalence of CA-MRSA strains isolated from a wider range of clinical specimen types. Second, MRSA detection does not necessarily correspond to clinical MRSA infection, as the specimens used in this study, apart from the blood specimens, were not sterile. However, as MRSA colonization usually precedes
MRSA infection, an overall increase in the proportion of CA-MRSA strains in clinical specimens has an adjunctive role in indicating an increase in the prevalence of CA-MRSA infection. Third, resistance to other antimicrobials, such as linezolid and tigecycline, may also be of interest [24], but was not investigated because it was not the focus of this study.

In conclusion, CA-MRSA strains were observed to be increasingly prevalent in a Korean hospital environment and to exhibit increased phenotypic diversity. Further genotypic studies on MRSA isolates are required to elucidate clonal changes in CAMRSA strains and the sequent clinical impact on healthcare services.

\section{ACKNOWLEDGEMENTS}

None.

\section{AUTHOR CONTRIBUTIONS}

Conceptualization: Choi SH; Methodology and software: Yu SH, Lee JH, Choi SH; Validation: Kim MC, Choi SH; Formal analysis: Choi SH; Investigation: Yu SH, Lee JH, Choi SH; Data curation: Yu SH, Lee JH, Choi SH; Writing-original draft: Yu SH, Lee JH, Choi SH; Writing-review \& editing: Kim MC, Chung JW, Choi SH, Lee MK; Visualization: Choi SH; Project administration: Choi SH.

\section{CONFLICTS OF INTEREST}

None declared.

\section{RESEARCH FUNDING}

None declared.

\section{ORCID}

So Hyun Yu https://orcid.org/0000-0001-9565-5672 Jeong Hyun Lee https://orcid.org/0000-0002-3525-1331 Min-Chul Kim Seong-Ho Choi Jin-Won Chung Mi-Kyung Lee https://orcid.org/0000-0003-4410-5608 https://orcid.org/0000-0001-8108-2412 https://orcid.org/0000-0003-4811-6056 https://orcid.org/0000-0003-1824-476X

\section{REFERENCES}

1. Stryjewski ME and Corey GR. Methicillin-resistant Staphylococcus aure- 
us: an evolving pathogen. Clin Infect Dis 2014;58:S10-9.

2. David MZ and Daum RS. Community-associated methicillin-resistant Staphylococcus aureus: epidemiology and clinical consequences of an emerging epidemic. Clin Microbiol Rev 2010;23:616-87.

3. Kim ES, Song JS, Lee HJ, Choe PG, Park KH, Cho JH, et al. A survey of community-associated methicillin-resistant Staphylococcus aureus in Korea. J Antimicrob Chemother 2007;60:1108-14.

4. Seybold U, Kourbatova EV, Johnson JG, Halvosa SJ, Wang YF, King MD, et al. Emergence of community-associated methicillin-resistant Staphylococcus aureus USA300 genotype as a major cause of healthcare-associated bloodstream infections. Clin Infect Dis 2006;42:647-56.

5. Souli M, Ruffin F, Choi SH, Park LP, Gao S, Lent NC, et al. Changing characteristics of Staphylococcus aureus bacteremia: results from a 21year, prospective, longitudinal study. Clin Infect Dis 2019;69:1868-77.

6. Joo EJ, Chung DR, Ha YE, Park SY, Kang SJ, Kim SH, et al. Community-associated Panton-Valentine leukocidin-negative meticillin-resistant Staphylococcus aureus clone (ST72-MRSA-IV) causing healthcare-associated pneumonia and surgical site infection in Korea. J Hosp Infect 2012;81:149-55

7. Park SY, Chung DR, Yoo JR, Baek JY, Kim SH, Ha YE, et al. Sequence type 72 community-associated meticillin-resistant Staphylococcus aureus emerged as a predominant clone of nasal colonization in newly admitted patients. J Hosp Infect 2016;93:386-9.

8. Joo EJ, Chung DR, Kim SH, Baek JY, Lee NY, Cho SY, et al. Emergence of community-genotype methicillin-resistant Staphylococcus aureus in Korean hospitals: clinical characteristics of nosocomial infections by community-genotype strain. Infect Chemother 2017;49:109-16.

9. Joo EJ, Chung DR, Ha YE, Park SY, Kim HA, Lim MH, et al. Clinical predictors of community-genotype ST72-methicillin-resistant Staphylococcus aureus-SCCmec type IV in patients with community-onset S. aureus infection. J Antimicrob Chemother 2012;67:1755-9.

10. Shin E, Hong H, Park J, Oh Y, Jung J, Lee Y. Characterization of Staphylococcus aureus faecal isolates associated with food-borne disease in Korea. J Appl Microbiol 2016;121:277-86.

11. Lee J, Sung JY, Kim YM, Oh CE, Kim HB, Choi EH, et al. Molecular characterization of methicillin-resistant Staphylococcus aureus obtained from the anterior nares of healthy Korean children attending daycare centers. Int J Infect Dis 2011;15:e558-63.

12. Ko KS, Lee JY, Baek JY, Peck KR, Rhee JY, Kwon KT, et al. Characterization of Staphylococcus aureus nasal carriage from children attending an outpatient clinic in Seoul, Korea. Microb Drug Resist 2008;14:37-44.

13. Sung JY, Lee J, Choi EH, Lee HJ. Changes in molecular epidemiology of community-associated and health care-associated methicillin-resistant
Staphylococcus aureus in Korean children. Diagn Microbiol Infect Dis 2012;74:28-33.

14. Kim ES, Song JS, Lee HJ, Choe PG, Park KH, Cho JH, et al. A survey of community-associated methicillin-resistant Staphylococcus aureus in Korea. J Antimicrob Chemother 2008;62:214.

15. Peck KR, Baek JY, Song JH, Ko KS. Comparison of genotypes and enterotoxin genes between Staphylococcus aureus isolates from blood and nasal colonizers in a Korean hospital. J Korean Med Sci 2009;24:58591.

16. Bae IG, Kim JS, Kim S, Heo ST, Chang C, Lee EY. Genetic correlation of community-associated methicillin-resistant Staphylococcus aureus strains from carriers and from patients with clinical infection in one region of Korea. J Korean Med Sci 2010;25:197-202.

17. Moon SY, Lee HJ, Lee MS. Molecular characteristics of methicillin-resistant Staphylococcus aureus blood isolates: clonal spread of staphylococcal cassette chromosome mec type IVA between the community and the hospital. Microb Drug Resist 2010;16:217-22.

18. Kwon JC, Kim SH, Park SH, Choi SM, Lee DG, Choi JH, et al. Molecular epidemiologic analysis of methicillin-resistant Staphylococcus aureus isolates from bacteremia and nasal colonization at 10 intensive care units: multicenter prospective study in Korea. J Korean Med Sci 2011;26:60411.

19. Park KH, Chong YP, Kim SH, Lee SO, Choi SH, Lee MS, et al. Community-associated MRSA strain ST72-SCCmecIV causing bloodstream infections: clinical outcomes and bacterial virulence factors. J Antimicrob Chemother 2015;70:1185-92.

20. Bae E, Kim CK, Jang JH, Sung H, Choi Y, Kim MN. Impact of community-onset methicillin-resistant Staphylococcus aureus on Staphylococcus aureus bacteremia in a central Korea Veterans Health Service hospital. Ann Lab Med 2019;39:158-66.

21. Park SH, Park C, Yoo JH, Choi SM, Choi JH, Shin HH, et al. Emergence of community-associated methicillin-resistant Staphylococcus aureus strains as a cause of healthcare-associated bloodstream infections in Korea. Infect Control Hosp Epidemiol 2009;30:146-55.

22. Choi SH, Lee J, Jung J, Kim ES, Kim MJ, Chong YP, et al. A longitudinal study of adult patients with Staphylococcus aureus bacteremia over 11 years in South Korea. J Korean Med Sci [in press].

23. CLSI. Performance standards for antimicrobial susceptibility testing. 25th ed. CLSI supplement M100-S25. Wayne, PA: Clinical and Laboratory Standards Institute. 2015.

24. Yoo IY, Kang OK, Shim HJ, Huh HJ, Lee NY. Linezolid resistance in methicillin-resistant Staphylococcus aureus in Korea: high rate of false resistance to linezolid by the VITEK 2 system. Ann Lab Med 2020;40:57-62. 
Supplemental Data Table S1. Antimicrobial resistance patterns of MLST ST72-methicillin-resistant Staphylococcus aureus clinical isolates in Korea

\begin{tabular}{|c|c|c|c|c|c|c|c|c|c|}
\hline \multirow{2}{*}{ Reference } & \multirow{2}{*}{ Total (N) } & \multicolumn{8}{|c|}{ Antimicrobial resistance (N) } \\
\hline & & ERY & CLI & $\mathrm{CIP}$ & GEN & Fusidic acid & TET & RIF & TMP_SMX \\
\hline Kim, et al. 2008 [14] & 25 & 14 & 14 & 0 & 2 & & 1 & 0 & 0 \\
\hline Moon, et al. 2010 [17] & 2 & 1 & 1 & 0 & 0 & & 0 & 0 & 0 \\
\hline Lee, et al. 2011 [11] & 29 & 18 & 18 & & & & 0 & & \\
\hline Kwon, et al. 2011 [18] & 58 & 45 & 44 & & & 39 & & & \\
\hline Jo0, et al. 2012 [9] & 84 & 38 & 21 & & & 2 & & & \\
\hline Park, et al. 2015 [19] & 79 & 28 & 18 & 5 & 12 & 0 & & 0 & 0 \\
\hline Shin, et al. 2016 [10] & 36 & 16 & 0 & 1 & 11 & & 5 & & \\
\hline Bae, et al. 2019 [20] & 16 & 4 & 4 & 4 & 4 & 2 & 1 & & \\
\hline Choi, et al. (Unpublished data) [22] & 299 & 83 & 61 & 34 & 47 & 8 & 9 & 6 & 2 \\
\hline Overall resistance, $\mathrm{N}(\%)$ & 628 & $\begin{array}{c}200 / 559 \\
(35.78)\end{array}$ & $\begin{array}{c}182 / 628 \\
(28.98)\end{array}$ & $\begin{array}{l}88 / 515 \\
(17.09)\end{array}$ & $\begin{array}{l}76 / 457 \\
(16.63)\end{array}$ & $\begin{array}{c}12 / 478 \\
(2.51)\end{array}$ & $\begin{array}{l}55 / 459 \\
(11.98)\end{array}$ & $\begin{array}{l}6 / 405 \\
(1.48)\end{array}$ & $\begin{array}{l}2 / 405 \\
(0.49)\end{array}$ \\
\hline
\end{tabular}

Abbreviations: MLST, multilocus sequence typing; ST, sequence type; CLI, clindamycin; ERY, erythromycin; CIP, ciprofloxacin; GEN, Gentamycin; TET, Tetracycine; RIF, Rifampicin; TMP_SMX, trimethoprim/sulfamethoxazole. 